KEK-TH-1191

\title{
Superstring vertex operators in type IIB matrix model
}

\author{
Yoshihisa Kitazawa ${ }^{1,2, \text {, }}$ and Satoshi Nagaoka ${ }^{1, \text { 田 }}$ \\ ${ }^{1}$ High Energy Accelerator Research Organization (KEK), Tsukuba, Ibaraki 305-0801, Japan \\ ${ }^{2}$ Department of Particle and Nuclear Physics The Graduate University for Advanced Studies Tsukuba Ibaraki 305-0801 Japan
}

(Dated: published 20 June 2008)

\begin{abstract}
We clarify the relation between the vertex operators in type IIB matrix model and superstring. Green-Schwarz light-cone closed superstring theory is obtained from IIB matrix model on two dimensional noncommutative backgrounds. Superstring vertex operators should be reproduced from those of IIB matrix model through this connection. Indeed, we confirm that supergravity vertex operators in IIB matrix model on the two dimensional backgrounds reduce to those in superstring theory. Noncommutativity plays an important role in our identification. Through this correspondence, we can reproduce superstring scattering amplitudes from IIB matrix model.
\end{abstract}

\section{INTRODUCTION}

String theory is perturbatively reproduced from type IIB matrix model [1] via two dimensional noncommutative (NC) $\mathcal{N}=8$ supersymmetric gauge theory [2, 3, 4] in the IR limit [5]. The emergent string theory is GreenSchwarz (GS) type IIA superstring theory with light-cone gauge [6], which is also derived from commutative $\mathcal{N}=8$ super Yang-Mills 7].

In the two dimensional gauge theory, the IR limit corresponds to the strong coupling limit since the gauge coupling has dimension (length) ${ }^{-1}$. Dijkgraaf, Verlinde and Verlinde have shown that the IR limit corresponds to the free string limit. This logic is applicable to our case and actually we have derived the free Green-Schwarz string action from $\mathrm{NC}$ gauge theory by taking the strong coupling limit [5]. We emphasize here that the strong coupling limit (the IR limit) of NC gauge theory is the free string limit, not the low energy limit of Green-Schwarz string theory.

The winding number $w$ is reinterpreted as a light-cone momentum $p_{+}$in a T-dual interpretation. In NC gauge theory, such a duality is realized by identifying the momentum with the longitudinal coordinates. In our procedure, noncommutativity $\theta$ plays a crucial role to reproduce the worldsheet action. It is identified with the string scale $\alpha^{\prime}$ in the process of derivation.

Noncommutativity in $\mathrm{NC}$ gauge theory gives rise to various novel properties in comparison with the commutative gauge theory. Among them, we quote the following two aspects. The first property is the regularization of UV divergence, which gives rise to the UV/IR mixing effect [8]. The qualitative behavior of the correlation functions, such as the power of the momentum dependence, is affected by the existence of noncommutativity. The second property is the introduction of the scale in the theory. Since there are no scale parameters in the

*Electronic address: kitazawa@post.kek.jp

${ }^{\dagger}$ Electronic address: nagaoka@post.kek.jp action of IIB matrix model, the noncommutativity could provide a fundamental scale in the emergent theory. We have indeed identified the scale in NC gauge theory with the string scale in the previous paper [5]. Since the NC scale sets the eigenvalue density of the matrices, our identification of the string scale is consistent with [9].

In order to reveal the perturbative superstring picture in IIB matrix model more explicitly, it is important to demonstrate the procedure to calculate the superstring scattering amplitude from IIB matrix model. Since there is an open/closed duality in string theory, closed string theory will be naturally included in IIB matrix model. The massless sector of type II closed superstring consists of the supergravity multiplet. Supergravity controls the behavior of long range forces. The coupling between the fields in the supergravity multiplet and the operators in IIB matrix model has been clarified through the construction of the relevant vertex operators. The vertex operators for the supergravity multiplet are determined uniquely by the maximal $\mathcal{N}=2$ supersymmetry transformation in IIB matrix model [10, 11, 12]. These operators are constructed in [10] in the first study, where the wave functions are introduced as the representations of supergravity multiplet. In [11], the vertex operators are investigated further by expanding the supersymmetric Wilson loop operators [13]. In [12], the vertex operators are constructed up to the six-th order of MajoranaWeyl spinor $\lambda$. Since the algebraic calculation is very complicated, the complete structure of the vertex operators is not yet determined, but the vertex operators for the conjugate gravitino and two-form antisymmetric field are completely determined.

In this paper, we compare the IIB matrix model vertex operators on the two dimensional backgrounds with those in superstring. As we have derived supersymmetry transformation of GS light-cone superstring from IIB matrix model on the two dimensional backgrounds [5], we can reconstruct the superstring vertex operators based on the symmetry. In such a sense, this comparison can be regarded as a consistency check of the IIB matrix model vertex operator construction. Through this correspondence, we can reproduce the physical superstring 
scattering amplitudes from IIB matrix model.

In section II A, we review the supergravity vertex operators in IIB matrix model, which was constructed in [12]. In section IIB we review the closed superstring vertex operator construction of GS light-cone superstring theory. In section IC, we review the derivation of GS lightcone superstring action from IIB matrix model, which is carried out in the previous paper [5]. The derivation of supersymmetry transformation for GS light-cone superstring from IIB matrix model is also shown. The main investigation is carried out in section [II] where the vertex operators of IIB matrix model on the two dimensional $\mathrm{NC}$ backgrounds are analyzed. We verify that these operators are equivalent to superstring vertex operators. Section [IV is devoted to the conclusion. Light-cone open superstring vertex operators are shown in appendix A. In the appendix B, we construct type IIA closed string states in a radial quantization procedure. By this construction, we can directly calculate the scattering amplitude from IIB matrix model.

\section{VERTEX OPERATORS AND SUPERSYMMETRY TRANSFORMATION}

\section{A. Vertex operators in type IIB matrix model}

The Wilson loops are the vertex operators in IIB matrix model [14]. The gauge invariant observables in noncommutative gauge theory are the Wilson lines [15, 16, 17] which are obtained from the Wilson loops in matrix models. The behavior of closed string modes can be read from the correlation functions between the Wilson lines. The various properties of NC gauge theory are investigated so far, especially on homogeneous spaces 18, 19, 20]. In four dimensional backgrounds, the behavior of graviton propagators is investigated in detail through the vertex operators [21].

The vertex operators for the supergravity multiplet are constructed in [12]. These operators are linearly coupled to the supergravity fields and related with each other through the supersymmetry transformation. The result which has been known up to now is summarized as follows. $A_{\mu}(\mu=0,1, \cdots, 9)$ and $\psi$ are $N \times N$ Hermitian matrices and $\psi$ is a ten dimensional Majorana-Weyl spinor.

- Vertex operator for dilaton $\Phi$ :

$$
V^{\Phi}=\operatorname{Str} e^{i k \cdot A}
$$

where the symmetric trace Str is defined as

$$
\mathrm{Str} \mathcal{O}_{1} \mathcal{O}_{2} e^{i k A} \equiv \int_{0}^{1} d \alpha \operatorname{tr} \mathcal{O}_{1} e^{i \alpha k A} \mathcal{O}_{2} e^{i(1-\alpha) k A}
$$

- Vertex operator for dilatino $\tilde{\Phi}$ :

$$
V^{\tilde{\Phi}}=\operatorname{Str} e^{i k \cdot A} \bar{\psi} .
$$

- Vertex operator for the second rank antisymmetric tensor field $B_{\mu \nu}$ :

$$
V_{\mu \nu}^{B}=\operatorname{Str} e^{i k \cdot A}\left(\frac{1}{16} \bar{\psi} \cdot \not k \Gamma_{\mu \nu} \psi-\frac{i}{2} F_{\mu \nu}\right)
$$

where

$$
F_{\mu \nu} \equiv\left[A_{\mu}, A_{\nu}\right]
$$

- Vertex operator for gravitino $\Psi_{\mu}$ :

$$
V_{\mu}^{\Psi}=\operatorname{Str} e^{i k \cdot A}\left(-\frac{i}{12}\left(\bar{\psi} \cdot \not k \Gamma_{\mu \nu} \psi\right)-2 F_{\mu \nu}\right) \cdot \bar{\psi} \Gamma^{\nu}
$$

- Vertex operator for graviton $h_{\mu \nu}$ :

$$
\begin{array}{r}
V_{\mu \nu}^{h}=\operatorname{Str} e^{i k \cdot A}\left(-\frac{1}{96} k^{\rho} k^{\sigma}\left(\bar{\psi} \cdot \Gamma_{\mu \rho}{ }^{\beta} \psi\right) \cdot\left(\bar{\psi} \cdot \Gamma_{\nu \sigma \beta} \psi\right)\right. \\
-\frac{i}{4} k^{\rho} \bar{\psi} \cdot \Gamma_{\rho \beta(\mu} \psi \cdot F_{\nu)}{ }^{\beta}+\frac{1}{2} \bar{\psi} \cdot \Gamma_{(\mu}\left[A_{\nu)}, \psi\right] \\
\left.+2 F_{\mu}{ }^{\rho} \cdot F_{\nu \rho}\right) .
\end{array}
$$

- Vertex operator for the fourth rank antisymmetric tensor $C_{\mu \nu \rho \sigma}$ :

$$
\begin{aligned}
& V_{\mu \nu \rho \sigma}^{C}=\operatorname{Str} e^{i k \cdot A}\left(\frac{i}{8 \cdot 4 !} k_{\alpha} k_{\gamma}\left(\bar{\psi} \cdot \Gamma_{[\mu \nu}^{\alpha} \psi\right) \cdot\left(\bar{\psi} \cdot \Gamma_{\rho \sigma]}^{\gamma} \psi\right)\right. \\
&+\frac{i}{3} \bar{\psi} \cdot \Gamma_{[\nu \rho \sigma}\left[\psi, A_{\mu]}\right]+ \frac{1}{4} F_{[\mu \nu} \cdot\left(\bar{\psi} \cdot \Gamma_{\rho \sigma]}^{\gamma} \psi\right) k_{\gamma} \\
&\left.-i F_{[\mu \nu} \cdot F_{\rho \sigma]}\right) .
\end{aligned}
$$

- Vertex operator for the conjugate gravitino $\Psi_{\mu}^{c}$ :

$$
\begin{aligned}
& V_{\mu}^{\Psi^{c}}= \\
& \operatorname{Str} e^{i k \cdot A}\left(-\frac{i}{2 \cdot 5 !} k^{\lambda} k^{\tau}\left(\bar{\psi} \cdot \Gamma_{\mu \lambda}{ }^{\sigma} \psi\right) \cdot\left(\bar{\psi} \cdot \Gamma_{\nu \tau \sigma} \psi\right) \cdot \bar{\psi} \Gamma^{\nu}\right. \\
& +\frac{1}{24} k^{\lambda}\left(\bar{\psi} \cdot \Gamma_{\lambda \mu \nu} \psi\right) \cdot \bar{\psi} \Gamma^{\nu} \Gamma_{\rho \sigma} \cdot F^{\rho \sigma} \\
& -\frac{1}{6} k^{\lambda}\left(\bar{\psi} \cdot \Gamma_{\lambda \alpha \beta} \psi\right) \cdot \bar{\psi} \Gamma^{\beta} \cdot F^{\alpha}{ }_{\mu} \\
& \left.+i \bar{\psi} \Gamma_{\mu}\left[A_{\nu}, \psi\right] \bar{\psi} \Gamma^{\nu}-i F_{\mu \nu} \cdot F_{\rho \sigma} \cdot \bar{\psi} \Gamma^{\nu} \Gamma^{\rho \sigma}\right) .
\end{aligned}
$$

- Vertex operator for the conjugate antisymmetric ten- 
sor field $B_{\mu \nu}^{c}$ :

$V_{\mu \nu}^{B^{c}}=\operatorname{Str} e^{i k \cdot A}$

$\left(-\frac{1}{8 \cdot 6 !} k^{\lambda} k^{\tau} k^{\alpha}\left(\bar{\psi} \cdot \Gamma_{\mu \lambda}{ }^{\sigma} \psi\right) \cdot\left(\bar{\psi} \cdot \Gamma_{\gamma \tau \sigma} \psi\right) \cdot\left(\bar{\psi} \cdot \Gamma^{\gamma}{ }_{\alpha \nu} \psi\right)\right.$

$+\frac{i}{64}\left(\bar{\psi} \cdot k \Gamma_{\mu \alpha} \psi\right) \cdot F^{\alpha \beta}\left(\bar{\psi} \cdot \not \Gamma_{\beta \nu} \psi\right)$

$+\frac{i}{16 \cdot 4 !}\left(\bar{\psi} \cdot k \Gamma_{[\mu \alpha} \psi\right) \cdot\left(\bar{\psi} \cdot \not k \Gamma^{\alpha \sigma} \psi\right) \cdot F_{\sigma \nu]}$

$-\frac{1}{32} \bar{\psi} \cdot \Gamma_{[\mu}\left[A^{\sigma}, \psi\right] \cdot\left(\bar{\psi} \cdot \not k \Gamma_{\sigma \nu]} \psi\right)$

$-\frac{1}{64}\left(\bar{\psi} \cdot k \Gamma_{[\mu \alpha} \psi\right) \cdot \bar{\psi} \Gamma^{\alpha}\left[A_{\nu]}, \psi\right]$

$+\frac{i}{4 ! \cdot 32} \Xi_{\mu \nu \alpha \beta \gamma} \cdot\left(\bar{\psi} \cdot \Gamma^{\alpha \beta \gamma} \psi\right)$

$-\frac{i}{64}\left[A_{\alpha}, F^{\alpha \tau}\right] \cdot\left(\bar{\psi} \cdot \Gamma_{\tau \mu \nu} \psi\right)$

$+\frac{1}{64}\left(\bar{\psi} \cdot \Gamma_{\mu \nu \rho \sigma \lambda \tau} \not k \psi\right) \cdot F^{\rho \sigma} \cdot F^{\lambda \tau}$

$+\frac{1}{16}\left(\bar{\psi} \cdot \Gamma_{\rho \sigma} k \psi\right) \cdot F^{\rho \sigma} \cdot F^{\mu \nu}$

$-\frac{1}{8}\left(\bar{\psi} \cdot \Gamma_{\rho \sigma} \not k \psi\right) \cdot F^{\mu \rho} \cdot F^{\nu \sigma}+\frac{1}{8}\left(\bar{\psi} \cdot \Gamma_{[\mu \sigma} k \psi\right) \cdot F^{\sigma \alpha} \cdot F_{\alpha \nu]}$

$-\frac{1}{32}\left(\bar{\psi} \cdot \Gamma^{\mu \nu} k \psi\right) \cdot F^{\rho \sigma} \cdot F_{\sigma \rho}+\frac{i}{4} \bar{\psi} \cdot \Gamma_{\mu \nu \alpha}\left[A_{\beta}, \psi\right] \cdot F^{\alpha \beta}$

$+\frac{i}{8} \bar{\psi} \cdot \Gamma_{\rho \sigma[\mu}\left[A_{\nu]}, \psi\right] \cdot F^{\rho \sigma}+\frac{i}{4} \bar{\psi} \cdot \Gamma_{(\mu}\left[A_{\rho)}, \psi\right] \cdot F^{\rho}{ }_{\nu}$

$-\frac{i}{4} \bar{\psi} \cdot \Gamma_{(\nu}\left[A_{\rho)}, \psi\right] \cdot F^{\rho \mu}$

$\left.-i F_{\mu \rho} \cdot F^{\rho \sigma} \cdot F_{\sigma \nu}+\frac{i}{4} F_{\mu \nu} \cdot F^{\rho \sigma} \cdot F_{\sigma \rho}\right)$,

where

$$
\Xi_{\mu \nu \rho \sigma \tau}=\left\{\psi_{\alpha}, \psi_{\beta}\right\}\left(\Gamma_{0} \Gamma_{\mu \nu \rho \sigma \tau}\right)_{\alpha \beta}
$$

The remaining vertex operators for the conjugate dilatino and dilaton are only partly known.
- Vertex operator for the conjugate dilatino $\tilde{\Phi}^{c}$ :

$$
\begin{aligned}
& V^{\tilde{\Phi}^{c}}=\operatorname{Str} e^{i k \cdot A} \\
& \left(\frac{1}{8 !}\left(\bar{\psi} \cdot \Gamma^{\alpha \gamma} k \psi\right) \cdot\left(\bar{\psi} \cdot \Gamma_{\gamma \delta} k \psi\right) \cdot\left(\bar{\psi} \cdot \Gamma^{\delta \beta} k \psi\right) \cdot \bar{\psi} \Gamma_{\alpha \beta}\right. \\
& -\frac{i}{2 \cdot 5 !} F^{\mu \alpha} \cdot\left(\bar{\psi} \cdot k \Gamma_{\alpha \beta} \psi\right) \cdot\left(\bar{\psi} \cdot \not k \Gamma^{\beta \nu} \psi\right) \cdot \bar{\psi} \Gamma_{\mu \nu} \\
& +\cdots-\frac{1}{8 \cdot 4 !} F^{\mu \nu} \cdot F^{\rho \sigma}\left(\bar{\psi} \cdot \Gamma_{\mu \nu \rho \sigma \lambda \alpha \beta} \psi\right) k^{\lambda} \cdot \bar{\psi} \Gamma^{\alpha \beta} \\
& -\frac{1}{12} F^{\mu \alpha} \cdot F_{\alpha \beta} \cdot\left(\bar{\psi} \cdot k \Gamma^{\beta \nu} \psi\right) \cdot \bar{\psi} \Gamma_{\mu \nu} \\
& -\frac{1}{24} F^{\mu \alpha} \cdot\left(\bar{\psi} \cdot k \Gamma_{\alpha \beta} \psi\right) \cdot F^{\beta \nu} \cdot \bar{\psi} \Gamma_{\mu \nu} \\
& -\frac{1}{48} F^{\rho \sigma} \cdot\left(\bar{\psi} \cdot k \Gamma_{\rho \sigma} \psi\right) \cdot F^{\mu \nu} \cdot \bar{\psi} \Gamma_{\mu \nu}+\cdots \\
& +\cdots+\frac{i}{24} \bar{\psi} \cdot \Gamma_{\mu \nu \rho \sigma \lambda} F^{\mu \nu} \cdot F^{\rho \sigma} \cdot F^{\lambda \tau} \\
& \left.+i \bar{\psi} \cdot \Gamma^{\mu \nu}\left(F_{\mu \rho} \cdot F^{\rho \sigma} \cdot F_{\sigma \nu}-\frac{1}{4} F^{\rho \sigma} \cdot F_{\sigma \rho} \cdot F_{\mu \nu}\right)\right) .
\end{aligned}
$$

- Vertex operator for the conjugate dilaton $\Phi^{c}$ :

$$
\begin{aligned}
& V^{\Phi^{c}}=\operatorname{Str} e^{i k \cdot A} \\
& \left(\frac{1}{8 \cdot 8 !}\left(\bar{\psi} \cdot \Gamma^{\alpha \gamma} k \psi\right) \cdot\left(\bar{\psi} \cdot \Gamma_{\gamma \delta} k \psi\right) \cdot\left(\bar{\psi} \cdot \Gamma_{\delta \beta} k \psi\right) \cdot\left(\bar{\psi} \cdot \Gamma_{\alpha \beta} k \psi\right)\right. \\
& +\cdots \\
& +\frac{i}{48}\left(\bar{\psi} \cdot \Gamma_{\mu \nu \rho \sigma \lambda \tau} k \psi\right) \cdot F^{\mu \nu} \cdot F^{\rho \sigma} \cdot F^{\lambda \tau} \\
& +\left[A_{\mu}, \bar{\psi}\right] \cdot \Gamma_{\rho \sigma} \Gamma_{\nu} \psi \cdot F^{\mu \nu} \cdot F^{\rho \sigma} \\
& +\frac{i}{2}\left(\bar{\psi} \cdot \Gamma^{\mu \nu} \not k \psi\right) \cdot\left(F_{\mu \rho} \cdot F^{\rho \sigma} \cdot F_{\sigma \nu}-\frac{1}{4} F^{\rho \sigma} \cdot F_{\sigma \rho} \cdot F_{\mu \nu}\right) \\
& \left.-\left(F_{\mu \nu} \cdot F^{\nu \rho} \cdot F_{\rho \sigma} \cdot F^{\sigma \mu}-\frac{1}{4} F_{\mu \nu} \cdot F^{\nu \mu} \cdot F_{\rho \sigma} \cdot F^{\sigma \rho}\right)\right)
\end{aligned}
$$

In these expressions, the Lorentz indices $\mu, \nu, \rho, \sigma, \lambda, \tau, \alpha, \beta, \gamma, \delta$ run over $0,1, \cdots, 9$.

\section{B. Vertex operators in superstring theory}

We review the construction of the vertex operators for Green-Schwarz light-cone superstring. In the early 1980s, Green and Schwarz investigated the light-cone gauge formalism of superstring theory. The vertex operators are constructed in their formalism and the tree and one loop amplitudes are calculated [6, 22, 23, 24].

The light-cone coordinates $\phi_{i}(\tau, \sigma)$ decompose into the sum of the right-moving and left-moving components $\phi_{i}^{R}(\tau-\sigma)+\phi_{i}^{L}(\tau+\sigma)$. Since the two sectors separately describe the Fock space of open string states, closed string states are given by the direct products of open string states. Open string vertex operators are shown in the appendix A. There are $16 \times 16=256$ massless 
states in type IIA(IIB) superstring theory. By the product of two $\mathrm{SO}(8)$ representations, or two super YangMills multiplets, they are written as (left mover $) \times($ right mover $)=\left(8_{v}+8_{c}\right) \times\left(8_{v}+8_{s}\right)$ in type IIA superstring and $\left(8_{v}+8_{s}\right) \times\left(8_{v}+8_{s}\right)$ in type IIB superstring. We focus on IIA superstring in what follows.

$\frac{8_{v} \times 8_{v} \text { sector }}{8_{v} \times 8_{v} \text { sector }}$

$\overline{8_{v} \times 8_{v} \text { sector }}$ is decomposed into

$$
8_{v} \times 8_{v}=[0]+[2]+(2)=1+28+35_{v},
$$

where [2] denotes the second rank antisymmetric tensor field $B_{i j}$ and (2) denotes the symmetric traceless tensor $h_{i j}$. [0] corresponds to the dilaton $\Phi$.

The vertex operator for the symmetric traceless tensor in type IIA superstring theory is given by ${ }^{1}$

$$
\begin{aligned}
\left(\mathcal{V}_{i j}\right)^{h}(k)= & -\frac{1}{4 \pi \alpha^{\prime}} \int d \tau d \sigma V_{(i}^{B}\left(\frac{1}{2} k, \tau-\sigma\right) V_{j)}^{B}\left(\frac{1}{2} k, \tau+\sigma\right) \\
= & -\frac{1}{4 \pi \alpha^{\prime}} \int d \tau d \sigma\left(\dot{\phi}_{R}^{(i} \dot{\phi}_{L}^{j)}-\frac{1}{8} \Gamma_{a b}^{(i l} s_{R}^{a} s_{R}^{b} k^{l} \dot{\phi}_{L}^{j)}\right. \\
& -\frac{1}{8} \Gamma_{\dot{a} \dot{b}}^{(j l} s_{L}^{\dot{a}} s_{L}^{\dot{b}} k^{l} \dot{\phi}_{R}^{i)} \\
& \left.+\frac{1}{64} \Gamma_{a b}^{(i l} s_{R}^{a} s_{R}^{b} k^{l} \Gamma_{\dot{a} \dot{b}}^{j) m} s_{L}^{\dot{a}} s_{L}^{\dot{b}} k^{m}\right) e^{i k \phi}, \quad \text { II.15) }
\end{aligned}
$$

where

$$
\begin{aligned}
& (\cdots)_{R} \equiv(\cdots)_{R}(\tau-\sigma), \\
& (\cdots)_{L} \equiv(\cdots)_{L}(\tau+\sigma) .
\end{aligned}
$$

$V_{i}^{B}$ and $V_{1 a}^{F}\left(V_{2 \dot{a}}^{F}\right)$ denote the bosonic and fermionic vertex operators. The explicit form of these operators is shown in the appendix A. The indices $i, j, l, m$ run over the transverse directions $2, \cdots, 9$. The vertex operator for the second rank antisymmetric tensor is given by

$$
\begin{aligned}
& \left(\mathcal{V}_{i j}\right)^{B}(k)= \\
& -\frac{1}{4 \pi \alpha^{\prime}} \int d \tau d \sigma V_{[i}^{B}\left(\frac{1}{2} k, \tau-\sigma\right) V_{j]}^{B}\left(\frac{1}{2} k, \tau+\sigma\right) .
\end{aligned}
$$

The vertex operator for dilaton is given by

$$
\begin{aligned}
\mathcal{V}^{\Phi}(k)= & -\frac{1}{4 \pi \alpha^{\prime}} \int d \tau d \sigma V_{i}^{B}\left(\frac{1}{2} k, \tau-\sigma\right) V_{i}^{B}\left(\frac{1}{2} k, \tau+\sigma\right) \\
= & -\frac{1}{4 \pi \alpha^{\prime}} \int d \tau d \sigma\left(\dot{\phi}_{R}^{i} \dot{\phi}_{L}^{i}-\frac{1}{8} \Gamma_{a b}^{i l} s_{R}^{a} s_{R}^{b} k^{l} \dot{\phi}_{L}^{i}\right. \\
& -\frac{1}{8} \Gamma_{\dot{a} \dot{b}}^{i l} s_{L}^{\dot{a}} s_{L}^{\dot{b}} k^{l} \dot{\phi}_{R}^{i} \\
& \left.+\frac{1}{64} \Gamma_{a b}^{i l} s_{R}^{a} s_{R}^{b} k^{l} \Gamma_{\dot{a} \dot{b}}^{i m} s_{L}^{\dot{a}} s_{L}^{\dot{b}} k^{m}\right) e^{i k \phi} .
\end{aligned}
$$

1 In order to distinguish the superstring vertex operators from the vertex operators in IIB matrix model, we denote the closed superstring vertex operators in calligraphic characters. $\frac{8_{c} \times 8_{s} \text { sector }}{T h e 8_{c} \times 8_{s} \text { section }}$

The $8_{c} \times 8_{s}$ sector is decomposed into

$$
8_{c} \times 8_{s}=[1]+[3]=8_{v}+56,
$$

where [1] and [3] denote the 1-form field $C_{1}^{i}$ and 3-form antisymmetric tensor field $C_{3}^{i j l}$.

The vertex operator for the third rank antisymmetric tensor is given by

$$
\begin{aligned}
& \left(\mathcal{V}_{i j l}\right)^{C_{3}}(k)=-\frac{1}{4 \pi \alpha^{\prime}} \int d \tau d \sigma \\
& \left(V_{1 a}^{F}\left(\frac{1}{2} k, \tau-\sigma\right)_{R} \Gamma_{a \dot{b}}^{i j l} V_{1 \dot{b}}^{F}\left(\frac{1}{2} k, \tau+\sigma\right)_{L}\right),
\end{aligned}
$$

where $\Gamma_{a b}^{i j l}$ is inserted to construct the irreducible tensor. The vertex operator for the R-R 1-form field is given by

$$
\begin{aligned}
& \left(\mathcal{V}_{i}\right)^{C_{1}}(k)=-\frac{1}{4 \pi \alpha^{\prime}} \int d \tau d \sigma \\
& \quad\left(V_{1 a}^{F}\left(\frac{1}{2} k, \tau-\sigma\right)_{R} \gamma_{a \dot{b}}^{i} V_{1 \dot{b}}^{F}\left(\frac{1}{2} k, \tau+\sigma\right)_{L}\right) .
\end{aligned}
$$

$\underline{8_{v} \times 8_{s} \text { and } 8_{c} \times 8_{v} \text { sectors }}$

The representation $8_{v} \times 8_{s}\left(8_{c} \times 8_{v}\right)$ is decomposed into

$$
\begin{aligned}
& 8_{v} \times 8_{s}=[1]+[3]=8_{c}+56_{s}, \\
& 8_{c} \times 8_{v}=[1]+[3]=8_{s}+56_{c} .
\end{aligned}
$$

The vertex operator for gravitino is given by

$$
\begin{aligned}
\left(\mathcal{V}_{i}\right)^{\Psi}(k)= & -\frac{1}{4 \pi \alpha^{\prime}} \int d \tau d \sigma \\
& \left(V_{1 a}^{F}\left(\frac{1}{2} k, \tau-\sigma\right)_{R} V_{i}^{B}\left(\frac{1}{2} k, \tau+\sigma\right)_{L}\right), \\
\left(\mathcal{V}_{i}\right)^{\Psi}(k)= & -\frac{1}{4 \pi \alpha^{\prime}} \int d \tau d \sigma \\
& \left(V_{i}^{B}\left(\frac{1}{2} k, \tau-\sigma\right)_{R} V_{1 \dot{a}}^{F}\left(\frac{1}{2} k, \tau+\sigma\right)_{L}\right) .
\end{aligned}
$$

The vertex operator for dilatino is given by

$$
\begin{aligned}
& \mathcal{V}^{\tilde{\Phi}}(k)=-\frac{1}{4 \pi \alpha^{\prime}} \int d \tau d \sigma \\
& \left(V_{1 a}^{F}\left(\frac{1}{2} k, \tau-\sigma\right)_{R} \gamma_{a \dot{a}}^{i} V_{i}^{B}\left(\frac{1}{2} k, \tau+\sigma\right)_{L}\right), \\
& \mathcal{V}^{\tilde{\Phi}}(k)=-\frac{1}{4 \pi \alpha^{\prime}} \int d \tau d \sigma \\
& \left(V_{i}^{B}\left(\frac{1}{2} k, \tau-\sigma\right)_{R} \gamma_{a \dot{a}}^{i} V_{1 \dot{a}}^{F}\left(\frac{1}{2} k, \tau+\sigma\right)_{L}\right) .
\end{aligned}
$$

\section{IIB matrix model and Green-Schwarz superstring}

The action of IIB matrix model is written as

$$
S=-\frac{1}{g^{2}} \operatorname{Tr}\left(\frac{1}{4}\left[A^{\mu}, A^{\nu}\right]\left[A_{\mu}, A_{\nu}\right]+\frac{1}{2} \bar{\psi} \Gamma^{\mu}\left[A_{\mu}, \psi\right]\right) .
$$


By expanding the matrices

$$
A_{\mu}=p_{\mu}+a_{\mu}
$$

around the two dimensional NC background,

$$
\left[p_{\mu}, p_{\nu}\right]=i \theta_{\mu \nu},
$$

we obtain two dimensional noncommutative gauge theory with $\mathcal{N}=8$ supersymmetry $[2,3,4]$

$$
\begin{aligned}
& S= \\
& -\frac{\theta}{8 \pi g^{2}} \int d^{2} x \operatorname{tr}\left(\left[D^{\tilde{\mu}}, D^{\tilde{\nu}}\right]\left[D_{\tilde{\mu}}, D_{\tilde{\nu}}\right]+2\left[D^{\tilde{\mu}}, \phi^{i}\right]\left[D_{\tilde{\mu}}, \phi_{i}\right]\right. \\
& \left.+\left[\phi_{i}, \phi_{j}\right]\left[\phi_{i}, \phi_{j}\right]+2 \bar{\psi} \Gamma^{\tilde{\mu}}\left[D_{\tilde{\mu}}, \psi\right]+2 \bar{\psi} \Gamma_{i}\left[\phi_{i}, \psi\right]\right)_{*},(\text { III. } 28)
\end{aligned}
$$

where $\tilde{\mu}, \tilde{\nu}=0,1$ and $i, j=2, \cdots, 9 .{ }^{2}$ Trace of the matrices is mapped into the integral of the functions as

$$
\operatorname{Tr} \rightarrow \frac{\theta}{2 \pi} \operatorname{tr} \int d^{2} x
$$

where tr is a trace over $U(n)$ gauge group. Noncommutative parameter $\theta$ is an off-diagonal matrix element of the matrix $\theta \equiv \theta_{01}$.

In the IR limit, we can identify the perturbative string spectrum. Firstly,

i) * product reduces to ordinary commutative product since higher derivatives in the product can be neglected. The action (II.28) becomes the commutative $\mathcal{N}=8 U(n)$ super Yang-Mills in this limit

$$
\begin{aligned}
S= & -\frac{\theta}{8 \pi g^{2}} \int d^{2} x \operatorname{tr}\left(F_{\tilde{\mu} \tilde{\nu}}^{2}+2\left(D_{\tilde{\mu}} \phi_{i}\right)^{2}+\left[\phi_{i}, \phi_{j}\right]\left[\phi_{i}, \phi_{j}\right]\right. \\
& \left.+2 \bar{\psi} \Gamma^{\tilde{\mu}} D_{\tilde{\mu}} \psi+2 \bar{\psi} \Gamma_{i}\left[\phi_{i}, \psi\right]\right) .
\end{aligned}
$$

This action includes 8 matrix scalar fields $\phi_{i}$ and 16 matrix spinor fields $\psi=\left(s^{a}, s^{\dot{a}}\right)$. These fields transform in $8_{v}, 8_{c}$ and $8_{s}$ representations of $\mathrm{SO}(8)$ group. The perturbative vacua of this action are represented by the diagonal matrices $\phi_{i}=\left(\phi_{\text {diag }}\right)_{i}$, which form the moduli space of this theory.

By assuming that all the eigenvalues of matrices do not coincide with each other at any points on the worldsheet, all excitations of the off-diagonal modes become massive. Then,

ii) only diagonal elements are relevant in the low energy limit since massless excitations come from the diagonal elements. The interaction terms $\left[\phi_{i}, \phi_{j}\right]\left[\phi_{i}, \phi_{j}\right]$ and $2 \bar{\psi} \Gamma_{i}\left[\phi_{i}, \psi\right]$ vanish since the diagonal components commute. The gauge fields on the worldsheet decouple from the other fields. It has been found that the IR limit corresponds to the free string limit [7]. It is therefore

\footnotetext{
2 The metric is Wick rotated into the Euclidean signature in order to make contact with NC gauge theory.
}

consistent to modify the short distance structure of their construction as we have introduced the noncommutativity.

We transform the worldsheet coordinates from $R^{2}$ to $R^{1} \times S^{1}$ as

$$
z \equiv x_{0}+i x_{1}=e^{\tau+i \sigma}
$$

By the rescaling

$$
\psi_{R} \rightarrow \frac{1}{\sqrt{z}} \psi_{R}, \quad \psi_{L} \rightarrow \frac{1}{\sqrt{z}} \psi_{L}
$$

we obtain an action for a single string with the winding number $w$ as a string may wind $w$ times in the $\sigma$ direction

$$
\begin{aligned}
& S=-\frac{\theta}{4 \pi g^{2}} \int_{0}^{\infty} d \tau \int_{0}^{2 \pi w} d \sigma \\
& \left(\left(\partial_{\tau} \phi_{i}\right)^{2}+\left(\partial_{\sigma} \phi_{i}\right)^{2}+\bar{\psi}\left(\Gamma^{+} \partial_{+}+\Gamma^{-} \partial_{-}\right) \psi\right)
\end{aligned}
$$

Since the rank of the gauge group is related with the winding number of the strings as $n=\sum_{i} w_{i}$, multiple strings are obtained in general. GS superstring action with light-cone gauge is obtained by identifying $\frac{\theta}{4 \pi g^{2}} \equiv$ $\frac{1}{4 \pi \alpha^{\prime}}$.

This action (II.33) is invariant under the supersymmetry transformation with 32 supercharges of type IIA string theory which originates from $\mathcal{N}=2$ supersymmetry transformation in IIB matrix model as follows. Supersymmetry transformation in IIB matrix model is written as

$$
\begin{aligned}
\delta^{(1)} \psi & =\frac{i}{2}\left[A_{\mu}, A_{\nu}\right] \Gamma^{\mu \nu} \epsilon, \\
\delta^{(1)} A_{\mu} & =i \bar{\epsilon} \Gamma_{\mu} \psi, \\
\delta^{(2)} \psi & =-\eta \\
\delta^{(2)} A_{\mu} & =0 .
\end{aligned}
$$

On the two dimensional background, this transformation reduces in the IR limit to

$$
\begin{aligned}
& \delta^{(1)} s_{a}=-\dot{\phi}^{i} \gamma_{a \dot{a}}^{i} \epsilon^{\dot{a}}, \quad \delta^{(1)} s_{\dot{a}}=-\dot{\tilde{\phi}}^{i} \gamma_{\dot{a} a}^{i} \epsilon^{a} \\
& \delta^{(1)} \phi_{i}=2\left(\bar{\epsilon}^{\dot{a}} \gamma_{a \dot{a}}^{i} s^{a}+\bar{\epsilon}^{a} \gamma_{\dot{a} a}^{i} s^{\dot{a}}\right), \\
& \delta^{(2)} s_{a}=-\eta^{a}, \quad \delta^{(2)} s_{\dot{a}}=-\eta^{\dot{a}} \\
& \delta^{(2)} \phi_{i}=0
\end{aligned}
$$

where we have redefined $\eta^{a} \rightarrow \eta^{a}+\theta \epsilon, \eta^{\dot{a}} \rightarrow \eta^{\dot{a}}-\theta \epsilon$ to absorb the constant shift. The factors $\sqrt{z}$ and $\sqrt{\bar{z}}$ are absorbed by the redefinition of $\epsilon$ and $\eta$. $\gamma_{a \dot{a}}^{i}$ are the Clebsch-Gordan coefficients among three inequivalent $\mathrm{SO}(8)$ representations. This transformation leaves the Green-Schwarz light-cone string action (I..33) invariant. 


\section{SUPERSTRING VERTEX OPERATORS IN TYPE IIB MATRIX MODEL}

In this section, we derive superstring vertex operators from those of IIB matrix model on two dimensional backgrounds in the IR limit. We verify the equivalence between our construction and the light-cone formalism (except for the dilaton and dilatino) in subsections 3.1,3.2 and 3.3. For the dilaton and dilatino operators, we can find a convincing correspondence between the both constructions in section 3.4.

\section{A. $8_{v} \times 8_{v}$ sector}

Graviton $h_{i j}$

The vertex operators for graviton in IIB matrix model is written as

$$
\begin{array}{r}
V_{\mu \nu}^{h}=\operatorname{Str} e^{i k \cdot A}\left(-\frac{1}{96} k^{\rho} k^{\sigma}\left(\bar{\psi} \cdot \Gamma_{\mu \rho}{ }^{\beta} \psi\right) \cdot\left(\bar{\psi} \cdot \Gamma_{\nu \sigma \beta} \psi\right)\right. \\
-\frac{i}{4} k^{\rho} \bar{\psi} \cdot \Gamma_{\rho \beta(\mu} \psi \cdot F_{\nu)}{ }^{\beta}+\frac{1}{2} \bar{\psi} \cdot \Gamma_{(\mu}\left[A_{\nu)}, \psi\right] \\
\left.+2 F_{\mu}{ }^{\rho} \cdot F_{\nu \rho}\right) \cdot
\end{array}
$$

In the two dimensional background (II.27), the vertex operators are written in terms of the fields $\phi^{i}, s^{a}$ and $s^{\dot{a}}$. Furthermore, only the diagonal components are relevant in the IR limit. The symmetric trace Str is mapped into the integral as

$$
\operatorname{Str} \rightarrow \frac{\theta}{2 \pi} \int_{0}^{\infty} d \tau \int_{0}^{2 \pi w} d \sigma|z|^{2}(\cdots)
$$

By the field redefinition

$$
s_{a} \rightarrow \frac{1}{\sqrt{w z}} s_{a}, \quad s_{\dot{a}} \rightarrow \frac{1}{\sqrt{w \bar{z}}} s_{\dot{a}}
$$

and the scaling

$$
\tau \rightarrow w \tau, \quad \sigma \rightarrow w \sigma
$$

we obtain the correspondence

$$
\operatorname{Str} \rightarrow \frac{\theta}{2 \pi} \int_{0}^{\infty} d \tau \int_{0}^{2 \pi} d \sigma(\cdots) .
$$

In order to confirm that matrix model vertex operators are equivalent to superstring vertex operators, we investigate the IR limit of the graviton vertex operators term by term.

(a) $F_{i}{ }^{\mu} \cdot F_{j \mu}$ term

The bosonic part of the graviton vertex operator is written as

$$
2 \operatorname{Str} e^{i k \cdot A} F_{i}{ }^{\mu} \cdot F_{j \mu}
$$

where we can assume that the graviton has a transverse polarization. In the two dimensional background, leading contribution in the low energy limit gives

$$
\operatorname{Str} e^{i k \cdot A} F_{(i}^{\mu} \cdot F_{j) \mu} \rightarrow \frac{\theta}{\pi} \int d^{2} x e^{i k \cdot \phi} \partial_{-} \phi_{R}^{(i} \partial_{+} \phi_{L}^{j)}
$$

where

$$
\partial_{ \pm} \equiv \partial_{\tau} \pm i \partial_{\sigma}
$$

Thus, the operator (III.6) reduces to the first term in (II.15).

(b) $-\frac{i}{4} k^{\rho} \bar{\psi} \Gamma_{\rho \beta(i} \psi F_{j)}{ }^{\beta}$ term

Since $\Gamma_{+}$and $\Gamma_{-}$act on the fermion as

$$
\begin{aligned}
\bar{\psi}_{R} \Gamma_{+i j} \psi_{R} & =i \Gamma_{a b}^{i j} s_{R}^{a} s_{R}^{b}, \\
\bar{\psi}_{R} \Gamma_{-i j} \psi_{R} & =0, \\
\bar{\psi}_{L} \Gamma_{+i j} \psi_{L} & =0, \\
\bar{\psi}_{L} \Gamma_{-i j} \psi_{L} & =i \Gamma_{\dot{a} \dot{b}}^{i j} s_{L}^{\dot{a}} s_{L}^{b},
\end{aligned}
$$

we obtain

$$
\begin{aligned}
& -\frac{i}{4} \operatorname{Str} e^{i k A} k^{\rho} \bar{\psi} \Gamma_{\rho \beta(i} \psi F_{j)}^{\beta} \\
& =\frac{i}{8 \pi} \theta \int d \tau d \sigma e^{i k \phi}\left(k^{l} \bar{\psi} \Gamma_{l-(i} \psi \dot{\phi}_{R}^{j)}+k^{l} \bar{\psi} \Gamma_{l+(i} \psi \dot{\phi}_{L}^{j)}\right) \\
& =-\frac{\theta}{8 \pi} \int d \tau d \sigma e^{i k \phi}\left(\Gamma_{a b}^{(i l} s_{R}^{a} s_{R}^{b} k^{l} \partial_{+} \phi_{L}^{j)}\right. \\
& \left.+\Gamma_{\dot{a} \dot{b}}^{(i l} s_{L}^{\dot{a}} s_{L}^{b} k^{l} \partial_{-} \phi_{R}^{j)}\right),(\text { III.10) }
\end{aligned}
$$

by the field redefinition (III.3). Thus, this term is equivalent to the second and third terms in (II.15).

(c) $-\frac{1}{96} k^{\rho} k^{\sigma}\left(\bar{\psi} \Gamma_{i \rho}{ }^{\beta} \psi\right)\left(\bar{\psi} \Gamma_{j \sigma \beta} \psi\right)$ term

This term reduces to

$$
\begin{aligned}
& -\frac{1}{192} \operatorname{Str} e^{i k A} k^{\rho} k^{\sigma}\left(\bar{\psi} \Gamma_{(i \rho}{ }^{\beta} \psi\right)\left(\bar{\psi} \Gamma_{j) \sigma \beta} \psi\right) \\
= & -\frac{\theta}{384 \pi} \int d \tau d \sigma e^{i k \phi}\left(k^{l} k^{m}\left(\bar{\psi} \Gamma_{(i l}{ }^{+} \psi\right)\left(\bar{\psi} \Gamma_{j) m}{ }^{-} \psi\right)\right. \\
& +k^{l} k^{m}\left(\bar{\psi} \Gamma_{(i l}{ }^{-} \psi\right)\left(\bar{\psi} \Gamma_{j) m}{ }^{+} \psi\right) \\
& +k^{l} k^{m}\left(\bar{\psi} \Gamma_{(i l}{ }^{n} \psi\right)\left(\bar{\psi} \Gamma_{j) m n} \psi\right) \\
& \left.+k^{-} k^{-}\left(\bar{\psi} \Gamma_{(i-}{ }^{n} \psi\right)\left(\bar{\psi} \Gamma_{j)-n} \psi\right)\right) \\
= & \frac{\theta}{384 \pi} \int d \tau d \sigma e^{i k \phi}\left(2 \Gamma_{a b}^{(i l} s_{R}^{a} s_{R}^{b} k^{l} \Gamma_{\dot{a} \dot{b}}^{j) m} s_{L}^{\dot{a}} s_{L}^{\dot{b}} k^{m}\right. \\
& +4 \Gamma_{a \dot{a}}^{(i l n} s_{R}^{a} s_{L}^{\dot{a}} k^{l} \Gamma_{b \dot{b}}^{j) m n} s_{R}^{b} s_{L}^{\dot{b}} k^{m} \\
& \left.+k^{-} k^{-} \Gamma_{a b}^{i n} s_{a} s_{b} \Gamma_{c d}^{m n} s_{c} s_{d}\right) \\
= & \frac{\theta}{64 \pi} \int d \tau d \sigma e^{i k \phi} \Gamma_{a b}^{(i l} s_{R}^{a} s_{R}^{b} k^{l} \Gamma_{\dot{a} \dot{b}}^{j) m} s_{L}^{\dot{a}} s_{L}^{\dot{b}} k^{m}, \text { (III.11) }
\end{aligned}
$$

which is equivalent to the last term in (II.15).

(d) $\frac{1}{2} \bar{\psi} \cdot \Gamma_{(i}\left[A_{j)}, \psi\right]$ term 
This term vanishes in the IR limit.

Next, let us consider the light cone momentum in the exponential factor $k \cdot \phi=k_{i} \phi_{i}-k^{-} \phi^{+}-k^{+} \phi^{-} .{ }^{3}$ Gauge fields exist in this factor as

$$
k^{-} \phi^{+}+k^{+} \phi^{-}=k^{-}\left(p^{+}+a^{+}\right)+k^{+}\left(p^{-}+a^{-}\right) .
$$

The correlation function between the gauge fields is given by

$$
\left\langle e^{i k_{1}^{-} a_{1}^{+}}\left(z_{1}\right) \cdot e^{\left.i k_{2}^{+} a_{2}^{-}\right)}\left(z_{2}\right)\right\rangle \sim\left|z_{1}-z_{2}\right|^{-\alpha^{\prime} k_{1}^{-} k_{2}^{+}},
$$

where $\alpha^{\prime}=g^{2} / \theta$. If we put together the correlation functions between the gauge fields and scalar fields, the momentum dependent power of (III.13) is summed up in the covariant form. It is because we started with the covariant IIB matrix model action.

On the other hand, in the light-cone gauge formalism, $k \cdot \phi=k_{i} \phi_{i}-k^{-} \phi^{+}=k_{i} \phi_{i}-k^{-} \alpha^{\prime}\left(p^{+} \tau+x^{+}\right)$where $k^{+}=$ 0 . After rotating $\tau \rightarrow-i \tau$, the light-cone momentum in the vertex operator gives a factor

$$
\begin{aligned}
e^{-\alpha^{\prime} k_{1}^{-} p^{+} \tau_{1}}\left|k_{2}\right\rangle & \sim e^{-\alpha^{\prime} \tau_{1} k_{1}^{-} k_{2}^{+}}\left|k_{2}\right\rangle \\
& \sim\left|z_{1}-z_{2}\right|^{-\alpha^{\prime} k_{1}^{-} k_{2}^{+}}\left|k_{2}\right\rangle
\end{aligned}
$$

to the scattering amplitude. This factor is also summed up in the covariant form if we put together the contribution from the transverse and longitudinal modes. Thus, the light-cone momentum contributes consistently to the amplitude in the both formalism.

In this way, through the calculations (a),(b),(c) and (d), we have verified that the graviton vertex operator in IIB matrix model (II.7) reduces in the IR limit to

$$
\begin{aligned}
V_{i j}^{h}= & \operatorname{Str} e^{i k \cdot A}\left(-\frac{1}{96} k^{\rho} k^{\sigma}\left(\bar{\psi} \cdot \Gamma_{i \rho}{ }^{\beta} \psi\right) \cdot\left(\bar{\psi} \cdot \Gamma_{j \sigma \beta} \psi\right)\right. \\
& -\frac{i}{4} k^{\rho} \bar{\psi} \cdot \Gamma_{\rho \beta(i} \psi \cdot F_{j)}{ }^{\beta}+\frac{1}{2} \bar{\psi} \cdot \Gamma_{(i}\left[A_{j)}, \psi\right] \\
& \left.+2 F_{i}{ }^{\rho} \cdot F_{j \rho}\right) \\
\rightarrow & \frac{\theta}{\pi} \int_{0}^{\infty} d \tau \int_{0}^{2 \pi} d \sigma e^{i k \cdot \phi}\left(\dot{\phi}_{R}^{(i} \dot{\phi}_{L}^{j)}-\frac{1}{8} \Gamma_{a b}^{(i l} s_{R}^{a} s_{R}^{b} k^{l} \dot{\phi}_{L}^{j)}\right. \\
& -\frac{1}{8} \Gamma_{\dot{a} \dot{b}}^{(j l} s_{L}^{\dot{a}} s_{L}^{\dot{b}} k^{l} \dot{\phi}_{R}^{i)} \\
& \left.+\frac{1}{64} \Gamma_{a b}^{(i l} s_{R}^{a} s_{R}^{b} k^{l} \Gamma_{\dot{a} \dot{b}}^{j) m} s_{L}^{\dot{a}} s_{L}^{\dot{b}} k^{m}\right) \\
= & \left(\mathcal{V}_{i j}\right)^{h},
\end{aligned}
$$

3 In the light-cone gauge evaluations, we put $k^{+}=0$ except for the initial and final states. which is the graviton vertex operator in type IIA superstring theory.

Second rank antisymmetric tensor $B_{i j}$

The vertex operator for $B_{i j}$ in type IIB matrix model is shown in (II.4) and (II.10). We will verify that (II.10) in the two dimensional background is equivalent to (II.17), which is $B_{i j}$ type superstring vertex operator. For the graviton vertex operators, we have explicitly checked the equivalence term by term. But since (II.10) consists of many terms compared to (II.7), we verify the equality in a systematic way.

The operator in IIB matrix model which reduces to $B_{i j}$ type $(1,1)$ operator should be either of the following four forms

$$
\begin{aligned}
& \left(\bar{\psi} \Gamma_{i \ldots \psi}\right)_{L}\left(\bar{\psi} \Gamma_{j \ldots \psi}\right)_{R} \cdots \\
& \left(\bar{\psi} \Gamma_{i \ldots \psi} \psi\right)_{L}\left(F_{j \ldots}\right)_{R} \cdots \\
& \left(F_{i \ldots} \cdots\right)_{L}\left(\bar{\psi} \Gamma_{j \ldots} \psi\right)_{R} \cdots \\
& \left(F_{i \ldots} \cdots\right)_{L}\left(F_{j \ldots}\right)_{R} \cdots
\end{aligned}
$$

They should not contain no extra dimensionful operators. The terms in (II.10) which are of the forms (III.16) are only the following three terms

$$
\begin{aligned}
& \operatorname{Str} e^{i k \cdot A}\left(\frac{i}{64}\left(\bar{\psi} \cdot \not k \Gamma_{\mu \alpha} \psi\right) \cdot F^{\alpha \beta}\left(\bar{\psi} \cdot \not k \Gamma_{\beta \nu} \psi\right)\right. \\
& +\frac{1}{8}\left(\bar{\psi} \cdot \Gamma_{\mu \sigma} \not k \psi\right) \cdot F^{\sigma \alpha} \cdot F_{\alpha \nu} \\
& \left.-i F_{\mu \rho} \cdot F^{\rho \sigma} \cdot F_{\sigma \nu}\right) .
\end{aligned}
$$

Other terms do not reduce to the $(1,1)$ operators and do not give any contributions to the amplitude in the IR limit. By the field redefinition (III.3) and the scaling (III.4), we find that these terms are equivalent to

$$
\begin{aligned}
& \frac{\theta}{\pi} \int d \tau d \sigma e^{i k \phi}\left(\partial_{-} \phi_{R}^{[i} \partial_{+} \phi_{L}^{j]}-\frac{1}{8} \Gamma_{a b}^{[i l} s_{R}^{a} s_{R}^{b} k^{l} \partial_{+} \phi_{L}^{j]}\right. \\
& -\frac{1}{8} \Gamma_{\dot{a} \dot{b}}^{[i l} s_{L}^{\dot{a}} \partial_{-} \phi_{R}^{j]} s_{L}^{\dot{b}} k^{l} \\
& \left.+\frac{1}{64} \Gamma_{a b}^{[i l} s_{R}^{a} s_{R}^{b} k^{l} \Gamma_{\dot{a} \dot{b}}^{j] m} s_{L}^{\dot{a}} s_{L}^{\dot{b}} k^{m}\right)
\end{aligned}
$$

which is indeed the $B_{i j}$ superstring vertex operator (II.17). Thus, we have verified that, in the two dimensional background, the antisymmetric two form vertex operator in IIB matrix model (III.10) reduces to

$$
\begin{aligned}
\frac{i}{\theta} V_{i j}^{B^{c}}= & \frac{\theta}{\pi} \int d \tau d \sigma e^{i k \phi}\left(\partial_{-} \phi_{R}^{[i} \partial_{+} \phi_{L}^{j]}\right. \\
& -\frac{1}{8} \Gamma_{a b}^{[i l} s_{R}^{a} s_{R}^{b} k^{l} \partial_{+} \phi_{L}^{j]}-\frac{1}{8} \Gamma_{\dot{a} \dot{b}}^{[i l} s_{L}^{\dot{a}} \partial_{-} \phi_{R}^{j]} s_{L}^{\dot{b}} k^{l} \\
& \left.+\frac{1}{64} \Gamma_{a b}^{[i l} s_{R}^{a} s_{R}^{b} k^{l} \Gamma_{\dot{a} \dot{b}}^{j] m} s_{L}^{\dot{a}} s_{L}^{\dot{b}} k^{m}\right) .
\end{aligned}
$$

Dilaton $\Phi$

Although the dilaton vertex operators in IIB matrix model are not completely determined yet, we can find 
a convincing correspondence between the matrix model vertex operators and the superstring vertex operators. We will discuss this consistency in section 3.4.

\section{B. $8_{c} \times 8_{s}$ sector}

Since we start from the type IIB supergravity multiplet, the R-R sector includes 2-form and 4-form fields. From them, we can obtain 1-form and 3-form fields by singling out the light-cone (-) direction.

The third rank antisymmetric tensor $C_{i j l}$

Vertex operator for the 4-form field in IIB matrix model is present in (II.8) as

$$
\begin{aligned}
& V_{\mu \nu \rho \sigma}^{C}=\operatorname{Str} e^{i k \cdot A}\left(\frac{i}{8 \cdot 4 !} k_{\alpha} k_{\gamma}\left(\bar{\psi} \cdot \Gamma_{[\mu \nu}^{\alpha} \psi\right) \cdot\left(\bar{\psi} \cdot \Gamma_{\rho \sigma]}^{\gamma} \psi\right)\right. \\
&+\frac{i}{3} \bar{\psi} \cdot \Gamma_{[\nu \rho \sigma}\left[\psi, A_{\mu]}\right]+\frac{1}{4} F_{[\mu \nu} \cdot\left(\bar{\psi} \cdot \Gamma_{\rho \sigma]}^{\gamma} \psi\right) k_{\gamma} \\
&\left.-i F_{[\mu \nu} \cdot F_{\rho \sigma]}\right) \cdot \quad(\text { III. } 20)
\end{aligned}
$$

We can obtain the operators for 4-form, 3-form and 2form fields from the vertex operator (III.20) as

$$
\begin{aligned}
& \left(V_{i j l m}\right)^{C}, \\
& \left(V^{-}{ }_{i j l}\right)^{C}, \\
& \left(V^{+}{ }_{i j l}\right)^{C}, \\
& \left(V^{+-}{ }_{i j}\right)^{C} .
\end{aligned}
$$

We will show that only the 3 -form (III.22) survives in the IR limit.

First of all, we discuss the 4 -form vertex operator (III.21). The first term in the operator (III.21) is decomposed into

$$
\begin{aligned}
& \operatorname{Str}^{i k \cdot A} \frac{i}{8 \cdot 4 !}\left(k_{n} k_{p}\left(\bar{\psi} \cdot \Gamma_{[i j}^{n} \psi\right) \cdot\left(\bar{\psi} \cdot \Gamma_{l m]}^{p} \psi\right)\right. \\
& +k_{+} k_{p}\left(\bar{\psi} \cdot \Gamma_{[i j}{ }^{+} \psi\right) \cdot\left(\bar{\psi} \cdot \Gamma_{l m]}^{p} \psi\right) \\
& +k_{-} k_{p}\left(\bar{\psi} \cdot \Gamma_{[i j}{ }^{-} \psi\right) \cdot\left(\bar{\psi} \cdot \Gamma_{l m]}^{p} \psi\right) \\
& +k_{n} k_{+}\left(\bar{\psi} \cdot \Gamma_{[i j}^{n} \psi\right) \cdot\left(\bar{\psi} \cdot \Gamma_{l m]}^{+} \psi\right) \\
& +k_{n} k_{-}\left(\bar{\psi} \cdot \Gamma_{[i j}^{n} \psi\right) \cdot\left(\bar{\psi} \cdot \Gamma_{l m]}^{-} \psi\right) \\
& \left.+2 k_{+} k_{-}\left(\bar{\psi} \cdot \Gamma_{[i j}{ }^{+} \psi\right) \cdot\left(\bar{\psi} \cdot \Gamma_{l m]}{ }^{-} \psi\right)\right) .
\end{aligned}
$$

The first term and the last term in (III.25) vanish because $i, j, l$ and $m$ are antisymmetric. Other terms do not contain $(1,1)$ operators in the IR limit. Thus, this term does not contribute to the amplitude. The second term in the 4-form (III.21) vanish in the IR limit since $\psi$ and $A_{i}$ commute. The third and fourth terms in (III.21) apparently do not contain $(1,1)$ operator in the IR limit. Thus, we have confirmed that 4 -form (III.21) does not contribute to the amplitude.
In order to obtain the 3 -form field, we put $\mu$ direction with the - direction. Then, the first, third and fourth terms in (III.22) vanish from the condition that the indices $i, j$ and $l$ are antisymmetrized. By redefining the field as (III.3) and (III.4), the third term in (III.22) reduces to

$$
\begin{aligned}
\left(V^{-}{ }_{i j l}\right)^{C_{4}} & =\operatorname{Str} e^{i k \cdot \phi} \frac{i}{3} \bar{\psi} \cdot \Gamma_{[i j l]}\left[\psi, A^{-}\right] \\
& =\frac{i \theta}{3 \pi} \int d \tau d \sigma e^{i k \phi} s_{R}^{a} \Gamma_{a \dot{b}}^{[i j l]} s_{L}^{b}|w z|\left[k \phi, p^{-}\right]_{*} \\
& =k^{-} \frac{\theta}{3 \pi} \int d \tau d \sigma s_{R}^{a} \Gamma_{a \dot{b}}^{[i j l]} s_{L}^{\dot{b}} e^{i k \phi} \frac{\left|p^{+}\right|}{\theta} \text { (III.26) }
\end{aligned}
$$

In the last line, we use the canonical commutation relation $\left[p^{+}, p^{-}\right]=\theta$. The initial state can be represented by a coherent state $\exp \left(k^{+} \hat{p}^{-}\right)|0\rangle$. Since $w \hat{z}^{+}=\hat{p}^{+} / \theta$ on a NC plane, $w \hat{z}^{+}$is fixed to be $w \hat{z}^{+}=\left|p^{+}\right| / \theta$. Semiclassically we also find that $w \hat{z}^{-}=\left|p^{+}\right| / \theta$ since $\left|p^{+}\right|$is real. In this way, we have obtained the 3 -form field in $8_{c} \times 8_{s}$ sector. Thus, we have verified that the vertex operator (III.22) reduces to the superstring vertex operator (II.20). In this reduction, fermions have dimension $\left(\frac{1}{2}, \frac{1}{2}\right)$ and the background $A^{-} \rightarrow p^{-}$is interpreted to have dimension $\left(\frac{1}{2}, \frac{1}{2}\right)$.

If we single out the opposite direction + , we obtain the 3 -form field $\left(V^{+}{ }_{i j l}\right)^{C}$. This operator vanishes

$$
\begin{aligned}
\left(V^{+}{ }_{i j l}\right)^{C_{4}} & =\operatorname{Str} e^{i k \cdot \phi} \frac{i}{3} \bar{\psi} \cdot \Gamma_{[i j l]}\left[\psi, A^{+}\right] \\
& =\frac{i \theta}{3 \pi} \int d \tau d \sigma e^{i k \phi} s_{R}^{a} \Gamma_{a \dot{b}}^{[i j l]} s_{L}^{\dot{b}}|w z|\left[k \phi, p^{+}\right]_{*} \\
& =0,
\end{aligned}
$$

since $k^{+}=0$.

Finally, by the properties that Lorentz indices are antisymmetric, one can confirm that 2-form (III.24) does not contribute to the amplitude.

In this way, we have verified that the 4 -form matrix model vertex operator (II.10) reduces to the 3 -form superstring vertex operator (III.20) by singling out the direction.

One form field $C_{i}$

From (II.10), we can obtain the operators for 2-form, 1 -form and scalar fields as

$$
\begin{gathered}
\left(V_{i j}\right)^{B}, \\
\left(V_{i}^{-}\right)^{B}, \\
\left(V_{i}^{+}\right)^{B}, \\
\left(V^{+-}\right)^{B} .
\end{gathered}
$$

2-form (III.28) reduces to $B_{i j}$ superstring vertex operator (II.17) as we have verified in the previous section. 
1-form (III.29) and (III.30) reduce to

$$
\begin{aligned}
\frac{i}{\theta}\left(V_{i}^{-}\right)^{B} & =\frac{-1}{2 \theta} \operatorname{Str} e^{i k \cdot A} \bar{\psi} \cdot \Gamma_{i \alpha}^{-}\left[A_{\beta}, \psi\right] \cdot F^{\alpha \beta} \\
& =-\frac{i}{2 \pi} \int d \tau d \sigma e^{i k \phi} s_{R}^{a} \gamma_{a \dot{b}}^{i} s_{L}^{\dot{b}}|w z|\left[p^{-}, k \phi\right]_{*} \\
& \left.=k^{-} \frac{\theta}{2 \pi} \int d \tau d \sigma e^{i k \cdot \phi} s_{R}^{a} \gamma_{a \dot{b}}^{i} s_{L}^{\dot{b}} \frac{\left|p^{+}\right|}{\theta}, \text { (III.3 } 32\right) \\
\frac{i}{\theta}\left(V_{i}^{+}\right)^{B} & =0 .
\end{aligned}
$$

Other terms in (III.29) do not contribute to the amplitude in the IR limit. One can also confirm that 0 -form (III.31) does not contribute to the amplitude. The operators in (II.4) do not contribute or they have the dimension $\left(\frac{1}{2}, \frac{1}{2}\right),(1,0)$ or $(0,1)$.

Thus, we have verified that matrix model vertex operator (II.10) reduces to the superstring vertex operator (II.21) by singling out the - direction.

\section{C. $8_{v} \times 8_{s}\left(8_{c} \times 8_{v}\right)$ sector}

Gravitino $\Psi_{i}$

The vertex operator for gravitino $\Psi_{\mu}$ in IIB matrix model is shown in (II.6) and (II.9). The vertex operator (II.6) is

$$
V_{\mu}^{\Psi}=\operatorname{Str} e^{i k \cdot A}\left(-\frac{i}{12}\left(\bar{\psi} \cdot \not k \Gamma_{\mu \nu} \psi\right)-2 F_{\mu \nu}\right) \cdot \bar{\psi} \Gamma^{\nu}
$$

By the field redefinition (III.3) and the scaling (III.4), it reduces to

$$
\begin{aligned}
V_{i}^{\Psi} & = \\
& -\frac{\theta}{2 \pi} \int d \tau d \sigma e^{i k \phi}\left(\left(-2 \dot{\phi}^{i}+\frac{1}{4} \Gamma_{a b^{i l}} s^{a} s^{b} k^{l}\right)_{R} s_{L}^{\dot{a}} \sqrt{w z}\right. \\
& \left.+\sqrt{w \bar{z}} s_{R}^{a}\left(-2 \dot{\phi}^{i}+\frac{1}{4} \Gamma_{\dot{a} \dot{b}}^{i l} s^{\dot{a}} s^{\dot{b}} k^{l}\right)_{L}\right) \\
& =\frac{\theta}{\pi} \int d \tau d \sigma e^{i k \phi}\left(\left(\dot{\phi}^{i}-\frac{1}{8} \Gamma_{a b}^{i l} s^{a} s^{b} k^{l}\right)_{R} \sqrt{\frac{p^{+}}{\theta}} s_{L}^{\dot{a}}\right. \\
& \left.+\sqrt{\frac{p^{+}}{\theta}} s_{R}^{a}\left(\dot{\phi}^{i}-\frac{1}{8} \Gamma_{\dot{a} \dot{b}}^{i l} s^{\dot{a}} s^{\dot{b}} k^{l}\right)_{L}\right) .
\end{aligned}
$$

In this way, we have shown that (II.6) is equivalent to the superstring vertex operator in (II.23). One can check that the matrix model vertex operators for the conjugate gravitino (II.9) do not contribute to the amplitude in the IR limit.

\section{Dilaton and dilatino}

\section{$\underline{\text { Dilaton } \Phi}$}

The dilaton vertex operator in IIB matrix model is shown in (II.1)

$$
\operatorname{Str} e^{i k \cdot A}
$$

and (II.13)

$$
\begin{aligned}
& V^{\Phi^{c}}=\operatorname{Str} e^{i k \cdot A} \\
& \left(\frac{i}{2}\left(\bar{\psi} \cdot \Gamma^{\mu \nu} k \psi\right) \cdot\left(F_{\mu \rho} \cdot F^{\rho \sigma} \cdot F_{\sigma \nu}-\frac{1}{4} F^{\rho \sigma} \cdot F_{\sigma \rho} \cdot F_{\mu \nu}\right)\right. \\
& -\left(F_{\mu \nu} \cdot F^{\nu \rho} \cdot F_{\rho \sigma} \cdot F^{\sigma \mu}\right. \\
& \left.\left.-\frac{1}{4} F_{\mu \nu} \cdot F^{\nu \mu} \cdot F_{\rho \sigma} \cdot F^{\sigma \rho}\right)+\cdots\right) .
\end{aligned}
$$

In the two dimensional background, by the field redefinition (III.3) and the scaling (III.4), the explicitly written terms in (III.37) reduce to

$$
\begin{gathered}
\frac{\theta}{\pi} \int d \tau d \sigma e^{i k \phi}\left(-\frac{1}{8} \Gamma_{a b}^{i l} s_{R}^{a} s_{R}^{b} k^{l} \partial_{+} \phi_{L}^{i}\right. \\
\left.-\frac{1}{8} \partial_{-} \phi_{R}^{i} \Gamma_{\dot{a} \dot{b}}^{i l} s_{L}^{\dot{a}} s_{L}^{\dot{b}} k^{l}+\partial_{-} \phi_{R}^{i} \partial_{+} \phi_{L}^{i}\right) .
\end{gathered}
$$

These terms are present in (II.18) and the relative numerical coefficients also agree. But the last term in the superstring vertex operator (II.18) is missing. This term may come from the undetermined terms in (II.13), for example,

$$
\operatorname{Str} e^{i k \cdot A}\left(\bar{\psi} \cdot \Gamma_{\lambda \rho} k \psi\right)\left(\bar{\psi} \cdot \Gamma^{\lambda \rho} \not k \psi\right) \cdot F^{\mu \nu} F_{\nu \mu}
$$

We also need to examine the other terms, that is, $(\cdots)$ in (III.37). Explicitly determined terms in the (conjugate) dilaton vertex operators in the paper [12] is (III.37) and

$$
\begin{aligned}
& \operatorname{Str} e^{i k \cdot A} \\
& \left(\frac{1}{8 \cdot 8 !}\left(\bar{\psi} \cdot \Gamma^{\alpha \gamma} \not k \psi\right) \cdot\left(\bar{\psi} \cdot \Gamma_{\gamma \delta} k \psi\right) \cdot\left(\bar{\psi} \cdot \Gamma_{\delta \beta} \not k \psi\right) \cdot\left(\bar{\psi} \cdot \Gamma_{\alpha \beta} \not k \psi\right)\right. \\
& +\frac{i}{48}\left(\bar{\psi} \cdot \Gamma_{\mu \nu \rho \sigma \lambda \tau} \not k \psi\right) \cdot F^{\mu \nu} \cdot F^{\rho \sigma} \cdot F^{\lambda \tau} \\
& \left.+\left[A_{\mu}, \bar{\psi}\right] \cdot \Gamma_{\rho \sigma} \Gamma_{\nu} \psi \cdot F^{\mu \nu} \cdot F^{\rho \sigma}\right) .
\end{aligned}
$$

Therefore, we should check the behavior of these terms. Since the total dimension of the first term in (III.40) is four, there are no $(1,1)$ operators in this term. In order to obtain $(1,1)$ operators from the second term in (III.40), we need to fix $F^{\mu \nu}=F^{\rho \sigma} \equiv F^{+-}$. Apparently, this term does not contribute to the amplitude since it vanishes by the $\Gamma_{+-+-}$projection. The last term in (III.40) does not survive by the similar reason. Unless at least two of $\rho, \sigma$ and $\nu$ are transverse indices, we cannot find the $(1,1)$ operator. But if we choose $\rho$ and $\sigma$ to be the transverse directions as $\rho=i$ and $\sigma=j$, total dimension of the operator becomes three. Thus, we have confirmed that the other terms than (III.37) which are explicitly constructed in [12] do not contribute to the amplitude. 
Although there are many unknown terms in the matrix model vertex operators (II.13), such operators should reduce to the $(1,1)$ operators in (II.18). We thus believe that all the terms boil down to the superstring vertex operators (II.18).

Dilatino $\tilde{\Phi}$

The vertex operator for $\Psi_{\mu}$ in type IIB matrix model is shown in (II.3) and (II.12). The dilatino vertex operator in (II.12) reduces to that in superstring as

$$
\begin{aligned}
& -\frac{1}{\theta^{2}} V^{\tilde{\Phi}^{c}} \\
& =-\frac{1}{\theta^{2}} \operatorname{Str} e^{i k \cdot A}\left(-\frac{1}{12} F^{\mu \alpha} \cdot F_{\alpha \beta} \cdot\left(\bar{\psi} \cdot k \Gamma^{\beta \nu} \psi\right) \cdot \bar{\psi} \Gamma_{\mu \nu}\right. \\
& \left.+i \bar{\psi} \cdot \Gamma^{\mu \nu}\left(F_{\mu \rho} \cdot F^{\rho \sigma} \cdot F_{\sigma \nu}-\frac{1}{4} F^{\rho \sigma} \cdot F_{\sigma \rho} \cdot F_{\mu \nu}\right)\right) \\
& =\frac{\theta}{\pi} \int d \tau d \sigma e^{i k \phi} \sqrt{\frac{p^{+}}{\theta}}\left(-\frac{1}{8} \Gamma_{b c}^{i j} s_{R}^{b} s_{R}^{c} k^{j} \Gamma_{a \dot{a}}^{i} s_{L}^{\dot{a}}\right. \\
& \left.+\dot{\phi}_{R} \Gamma_{a \dot{a}}^{i} s_{L}^{\dot{a}}+R \leftrightarrow L\right) .
\end{aligned}
$$

Other known terms do not contribute to the amplitude as they are not $(1,1)$ operators. In the same reasoning, matrix model vertex operator (II.3) does not contribute to the amplitude. Although there remain undetermined terms in dilatino vertex operators in IIB matrix model, we believe that complete set of the operators is equivalent to those in type IIA superstring theory.

\section{CONCLUSION}

We have constructed type IIA closed string vertex operators directly from IIB matrix model on the two dimensional noncommutative backgrounds. The vertex operators which couple to supergravity multiplet were determined up to the six-th order of Majorana-Weyl spinor $\lambda$ in IIB matrix model [12]. In our analysis, gravitino, graviton, the fourth rank antisymmetric tensor field and the second rank antisymmetric tensor fields which contains up to $6 \lambda$ 's, show the perfect agreement with the corresponding superstring vertex operators. In this comparison, the identification of noncommutative scale $\frac{1}{\theta}$ with string scale $\alpha^{\prime}$ has played an important role, which we have adopted in the process of deriving the action [5]. Originally, the scale in NC gauge theory is identified with string scale in the dual supergravity description $[20,25,26]$. Since we take the commutative limit in our formulation, the direct relation between UV finiteness of string scattering amplitude and the regularization of UV divergence which give rise to UV/IR mixing effect in noncommutative gauge theory is unclear. It is interesting to investigate the relation between them in our formulation. The results in this paper and the previous paper [5] are summarized in Figure 1. The process $(1)+(2)$ is described in [5]. In this paper, the relation (4) is explicitly demonstrated, which can be regarded as the confirmation of the other process, especially process (3). At the first sight, on two dimensional backgrounds of IIB matrix model, the vertex operators are extremely complicated. But supersymmetry restricts the possible terms and the operators relevant to the amplitudes become the same as the vertex operators in superstring theory. After identifying the physical states, we can calculate the multi-point scattering amplitude in a standard way.

We have not yet reproduced the complete vertex operators in IIB matrix model due to algebraic complexity. Since the complete forms of the conjugate dilatino and dilaton vertex operators are not yet known, we can not compare these operators. However, we have found all the necessary pieces of each GS superstring vertex operator in IIB matrix model vertex operators even in those cases.

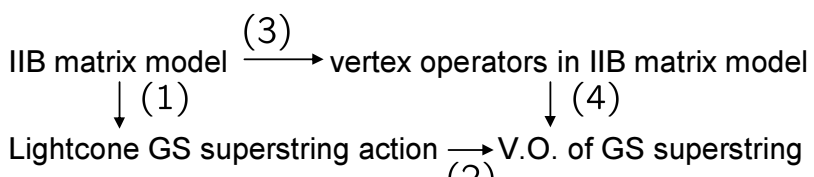

(2)

FIG. 1: Vertex operators for Green-Schwarz light-cone superstring are derived from IIB matrix model. The constructions $(1)+(2)$ and $(3)+(4)$ should give the same result.

\section{Acknowledgments}

This work is supported in part by the Grant-in-Aid for Scientific Research from the Ministry of Education, Science and Culture of Japan. The work of S.N. is supported in part by the Research Fellowship of the Japan Society for the Promotion of Science for Young Scientists.

\section{APPENDIX A: OPEN SUPERSTRING VERTEX OPERATORS}

The bosonic (vector) and fermionic (spinor) vertex operators of light-cone open superstring are written as

$$
\begin{aligned}
& V_{B}(\zeta, k)=\zeta^{\mu} V_{\mu}^{B}(k)=\left(\zeta^{i} B^{i}-\zeta^{-} B^{+}\right) e^{i k \cdot \phi}, \\
& V_{F}(u, k)=u^{a} V_{1 a}^{F}(k)+u^{\dot{a}} V_{2 \dot{a}}^{F}(k)=\left(u^{a} F_{1}^{a}+u^{\dot{a}} F_{2}^{\dot{a}}\right) e^{i k \cdot \phi} .
\end{aligned}
$$

where $B^{i}, B^{+}, F_{1}^{a}$ and $F_{2}^{\dot{a}}$ are written in terms of $\phi_{i}$ and $s^{a}$ as

$$
\begin{aligned}
B^{+} & =p^{+} \\
B^{i} & =\left(\dot{\phi}^{i}-R_{i j} k^{j}\right), \\
F_{2}^{\dot{a}} & =\left(2 p^{+}\right)^{-1 / 2}\left[(\Gamma \cdot \dot{\phi} s)^{\dot{a}}+\frac{1}{3}\left(\Gamma^{i} s\right)^{\dot{a}} R^{i j} k^{j}\right], \\
F_{1}^{a} & =\left(\frac{p^{+}}{2}\right)^{1 / 2} s^{a} .
\end{aligned}
$$


$s^{a}$ belong to $8_{s}$ representation in our convention. $R^{i j}(\tau)$ is defined by

$$
R^{i j}(\tau)=\frac{1}{4} \Gamma_{a b}^{i j} s^{a}(\tau) s^{b}(\tau),
$$

where

$$
\Gamma_{a b}^{i j} \equiv \frac{1}{2}\left(\gamma_{a \dot{a}}^{i} \gamma_{\dot{a} b}^{j}-\gamma_{a \dot{a}}^{j} \gamma_{\dot{a} b}^{i}\right)
$$

The matrices $\Gamma^{i}$ are represented in the 16-dimensional $\left(8_{s}+8_{c}\right)$ representation of spin (8) as

$$
\Gamma^{i}=\left(\begin{array}{cc}
0 & \gamma_{a \dot{a}}^{i} \\
\gamma_{\dot{b} b}^{i} & 0
\end{array}\right)
$$

We consider the operators which carry the momentum $k^{\mu}$ with $k^{+}=0,\left(k^{i}\right)^{2}=0$. $\left(\zeta^{+}, \zeta^{-}, \zeta^{i}\right)$ represents the wave function for the vector state, and $\left(u^{a}, u^{\dot{a}}\right)$ represents the wave function for the spinor state.

\section{APPENDIX B: TYPE IIA CLOSED STRING STATES IN TYPE IIB MATRIX MODEL}

In order to calculate the multi point superstring amplitude, we have to identify closed string states. Closed string states are constructed by the direct products of the left movers and right movers in string theory. In IIB matrix model on the two dimensional background, we can also construct closed string states in a radial quantization as the product of the separate states corresponding to the left movers and right movers, respectively.

Since the origin in the coordinate system $z$ becomes the infinite past in the conformal mapping (II.31), we can insert a local operator at the origin and obtain its charges by the appropriate contour integrals around the origin. The asymptotic states correspond to the local operators. 4

Before we define the massless ground states of closed strings, we define the massless ground states of open strings

$$
|i\rangle, \quad|\dot{a}\rangle
$$

as the states in the $8_{v}\left(8_{c}\right)$ representation of spin (8). They are normalized as

$$
\langle i \mid j\rangle=\delta_{i j},\langle\dot{a} \mid \dot{b}\rangle=\delta_{\dot{a} \dot{b}} .
$$

Any physical states $|\Lambda, k\rangle$ are obtained by inserting the vertex operators in the far past as

$$
|\Lambda, k\rangle=\lim _{\tau \rightarrow-\infty} e^{-\tau} V_{B}(k)|0,0\rangle .
$$

It is because zero mode operator $Z_{0}$ acts as

$$
\begin{aligned}
& Z_{0}|0,0\rangle \equiv e^{i k \cdot \phi} z^{k \cdot p+1}|0,0\rangle=z|0, k\rangle \\
& \langle 0,0| Z_{0} \equiv\langle 0,0| z^{k \cdot p-1} e^{i k \cdot x}=\frac{1}{z}\langle 0, k|
\end{aligned}
$$

The massless vector states transform as

$$
\begin{aligned}
R_{0}^{i j}|k\rangle & \equiv \frac{1}{4} s_{0}^{a} \Gamma_{a b}^{i j} s_{0}^{b}|k\rangle \\
& =\delta^{j k}|i\rangle-\delta^{i k}|j\rangle
\end{aligned}
$$

where $s_{0}$ is the zero mode of $s . R_{0}$ is the zero mode helicity operator. The massless spinor states transform as

$$
R_{0}^{i j}|\dot{a}\rangle=-\frac{1}{2} \Gamma_{\dot{a} \dot{b}}^{i j}|b\rangle
$$

The ground states are mapped to each other by the fermionic zero mode as

$$
\begin{aligned}
s_{0}^{a}|\dot{a}\rangle & =\frac{1}{\sqrt{2}} \Gamma_{a \dot{a}}^{i}|i\rangle, \\
s_{0}^{a}|i\rangle & =\frac{1}{\sqrt{2}} \Gamma_{a \dot{a}}^{i}|\dot{a}\rangle .
\end{aligned}
$$

A vector state $|\zeta\rangle$ is defined by

$$
|\zeta\rangle=|i\rangle \zeta^{i}
$$

and a spinor state $|u\rangle$ is defined by

$$
|u\rangle=|\dot{a}\rangle \frac{u^{\dot{a}}(k)}{\sqrt{k^{+}}} .
$$

Closed string states are constructed by the direct product of the left-movers and right-movers.
[1] N. Ishibashi, H. Kawai, Y. Kitazawa and A. Tsuchiya, "A Large-N Reduced Model as Superstring," Nucl. Phys. B498 (1997) 467, hep-th/9612115.

[2] A. Connes, M. Douglas and A. Schwarz, "Noncommuta- tive Geometry and Matrix Theory: Compactification on Tori," JHEP9802 (1998) 003, hep-th/9711162.

[3] H. Aoki, N. Ishibashi, S. Iso, H. Kawai, Y. Kitazawa and T. Tada, "Non-commutative Yang-Mills in IIB Matrix 
Model," Nucl. Phys. 565 (2000) 176, hep-th/9908141

[4] M. Li, "Strings from IIB Matrices," Nucl. Phys. B499 (1997) 149, hep-th/9612222.

[5] Y. Kitazawa and S. Nagaoka, "Green-Schwarz superstring from type IIB matrix model," Phys. Rev. D77 (2008) 026009, arXiv:0708.1077 hep-th].

[6] M. B. Green and J. H. Schwarz, "Supersymmetrical String Theories," Phys. Lett. B109 (1982) 444.

[7] R. Dijkgraaf, E. Verlinde and H. Verlinde, "Matrix String Theory," Nucl. Phys. B500 (1997) 43, hep-th/9703030

[8] S. Minwalla, M.V. Raamsdonk and N. Seiberg, "Noncommutative Perturbative Dynamics," JHEP 0002 (2000) 020, hep-th/9912072

[9] H. Kawai and M. Sato, "Perturbative Vacua from IIB Matrix Model," Phys. Lett. B659 (2008) 712, arXiv:0708.1732 hep-th].

[10] Y. Kitazawa, "Vertex Operators in IIB Matrix Model," JHEP 0204 (2002) 004, hep-th/0201218.

[11] S. Iso, H. Terachi and H. Umetsu, "Wilson Loops and Vertex Operators in Matrix Model," Phys. Rev. D70 (2004) 125005, hep-th/0410182.

[12] Y. Kitazawa, S. Mizoguchi and O. Saito, "Progress toward the Determination of Complete Vertex Operators for The IIB Matrix Model," Phys. Rev. D75 (2007) 106002, hep-th/0612080

[13] K. Hamada, "Supersymmetric Wilson Loops in IIB Matrix Model," Phys. Rev. D56 (1997) 7503, hep-th/9706187.

[14] M. Fukuma, H. Kawai, Y. Kitazawa and A. Tsuchiya, "String Field Theory from IIB Matrix Model," Nucl. Phys. B510 (1998) 158, hep-th/9705128

[15] N. Ishibashi, S. Iso, H. Kawai and Y. Kitazawa, "Wilson Loops in Non-commutative Yang-Mills," Nucl. Phys.
B573 (2000) 573, hep-th/9910004.

[16] D. J. Gross, A. Hashimoto and N. Itzhaki, "Observables of Non-Commutative Gauge Theories," Adv. Theor. Math. Phys. 4 (2000) 893, hep-th/0008075.

[17] A. Dhar and Y. Kitazawa, "High-Energy Behavior of Wilson Lines," JHEP 0102 (2001) 004, hep-th/0012170

[18] Y. Kitazawa, "Matrix Models in Homogeneous Spaces," Nucl. Phys. B642 (2002) 210, hep-th/0207115

[19] Y. Kitazawa, Y. Takayama and D. Tomino, "Correlators of Matrix Models on Homogeneous Spaces," Nucl. Phys. B700 (2004) 183, hep-th/0403242.

[20] Y. Kitazawa, Y. Takayama and D. Tomino, "Wilson Line Correlators in N=4 Non-commutative Gauge Theory on $S^{2} \times S^{2}$," Nucl. Phys. B715 (2005) 665, hep-th/0412312.

[21] Y. Kitazawa and S. Nagaoka, "Graviton Propagators on Fuzzy G/H," JHEP 0602 (2006) 001, hep-th/0512204

[22] M. B. Green and J. H. Schwarz, "Supersymmetrical Dual String Theory," Nucl. Phys. B181 (1981) 502.

[23] M. B. Green and J. H. Schwarz, "Supersymmetrical Dual String Theory. 2. Vertices and Trees," Nucl. Phys. B198 (1982) 252.

[24] M. B. Green and J. H. Schwarz, "Supersymmetrical Dual String Theory. 3. Loops and Renormalization," Nucl. Phys. B198 (1982) 441.

[25] N. Ishibashi, S. Iso, H. Kawai and Y. Kitazawa, "String Scale in Noncommutative Yang-Mills," Nucl. Phys. B583 (2000) 159, hep-th/0004038.

[26] Y. Kitazawa and S. Nagaoka, "Graviton Propagators in Supergravity and Noncommutative Gauge Theory," Phys. Rev. D75 (2007) 046007, hep-th/0611056 